This paper was published in the proceedings of SPIE Photonics West 2006, Vol. 6116-16 (2006), Integrated Optoelectronical Devices: Optical Components and Materials III, and is made available as an electronic preprint with permission of SPIE. One print or electronic copy may be made for personal use only. Systematic or multiple reproduction, distribution to multiple locations via electronic or other means, duplication of any material in this paper for a fee or for commercial purposes, or modification of the content of the paper are prohibited.

\title{
The Potential of Direct Nanoparticle Deposition for the Next Generation of Optical Fibers
}

\author{
Simo Tammela, Mikko Söderlund, Joona Koponen, Valery Philippov and Per Stenius \\ Liekki Corporation, Sorronrinne 9, FI-08500 LOHJA FINLAND
}

\begin{abstract}
Fiber lasers offer substantial advantages compared to conventional solid-state lasers due to their high efficiency, compact size, diffraction-limited beam quality, tunability, and facile thermal management. A number of important applications require high peak powers and pulse energies, which has generated great interest in Yb-doped, large-modearea (LMA) fibers. Liekki has pioneered a new manufacturing technology for rare-earth-doped fibers, Direct Nanoparticle Deposition (DND), that is capable of producing fibers uniquely well suited to power scaling. Conventional fiber fabrication methods are characterized by poor process accuracy and flexibility due to the large particle sizes and relatively small number of deposition layers (2-10). In contrast, DND provides independent control of the composition of hundreds of layers that make up the core, thereby allowing previously unattainable precision, accuracy, and uniformity in the index and rare-earth-dopant profiles. DND allows the simultaneous use of both gasphase and liquid precursors, providing unprecedented flexibility in the glass composition. Furthermore, DND enables fabrication of fibers with extremely high rare-earth concentrations, which minimizes the required fiber length and correspondingly raises the threshold power for nonlinear processes. Finally, the single-step, direct-deposition process makes manufacturing of fibers rapid and cost-effective, even for fibers with large core diameters or sophisticated geometries and dopant distributions. DND fibers have shown high conversion efficiency (low clustering), low photodarkening, and high damage threshold. DND thus promises to revolutionize the use of fiber lasers in applications previously restricted to bulk, solid-state lasers and to enable new applications of high-power lasers.
\end{abstract}

Keywords: Fiber laser, Rare-earth glass, Fiber production, Active fiber

\section{INTRODUCTION}

Double clad fiber based systems have become increasingly popular because of the high output beam quality and brightness, the possibility to use low brightness pump sources, and the high area to volume ratio which helps to mitigate the heat generated in the fiber ${ }^{1}$. Advances in design and manufacturing of highly $\mathrm{Yb}$-doped double cladding fibers have enabled the realization of high-average-power diffraction-limited fiber amplifiers reaching $\mathrm{mJ}$ pulse energies and MW peak powers, as well as continuous wave fiber lasers exceeding $1 \mathrm{~kW}$ output powers with nearly single-mode output beams $^{2,3,4,5,6,7,8}$.

The Direct Nanoparticle Deposition (DND) technology, owned and developed by Liekki, is a completely new fiber manufacturing technology, ideally suited for the demanding needs of advanced fiber laser applications. DND provides the flexibility to engineer the glass matrix into which the rare-earth ions are dissolved. This capability makes it possible to increase the rare-earth concentration without sacrificing the fiber performance with effects resulting from too high local rare-earth ion concentration. Furthermore, DND technology inherently provides radial control of dopants, resulting in excellent flatness of the refractive index profile, a key attribute in large, low numerical aperture cores. This ability also facilitates the fabrication of advanced waveguiding structures in which the gain provided by rare-earth ion is independently controlled. By adjusting the rare-earth ion concentration profile, it is possible to provide more gain to the fundamental mode with respect to higher order modes guided by the waveguide, thus helping in achieving diffractionlimited output even from very large, highly multimode waveguide structures. A further advantage of DND technology arises from the ability to fabricate large diameter core performs. This feature is directly compatible with cost-efficient and rapid manufacturing LMA fibers with large cores and large core-to-cladding ratios. Large diameter core preforms also simplifies the Panda type PM fiber production, as it is more practical to drill short, large diameter holes.

Finally, the ability of the DND process to change the glass composition easily, the rapid development cycle and the inherent uniformity has enabled systematic research on photodarkening, one of the key limiting factors on the reliability of Yb-doped fiber lasers and amplifiers. This research has relieved that the amount photodarkening depends on glass composition and that the photodarkening rate has an approximately seventh-order dependence on the inversion level of 
the Yb-ions. These relations can be used to develop more accurate measurements for photodarkening, to forecast the behavior of the Yb-doped fibers in different applications, as well as to understand the physical mechanism behind the photodarkening process in Yb-fibers.

\section{DND PROCESS}

\subsection{Conventional rare-earth doping processes}

The main challenge in manufacturing rare earth doped fibers has been the search for glass forming process, which can handle the low vapor pressure raw materials like rare-earth halides. The Modified Chemical Vapor Deposition (MCVD) and solution doping with liquid rare earth ions, the dominant technology used today, was developed for the telecom needs with small cores $(3-4 \mu \mathrm{m})$ and low doping ${ }^{10}$. In this process a porous layer of silica glass is produced on the inner side of a quartz tube. The tube is soaked into water containing rare-earth salts, like $\mathrm{YbCl}_{3}$. After drying the porous layer including the chloride with its crystalline water $\left(\mathrm{YbCl}_{3} 6\left(\mathrm{H}_{2} \mathrm{O}\right)\right)$ is heated, and the ytterbium chlorine forms hydroxide $\left(\mathrm{Yb}(\mathrm{OH})_{3}\right)$ and hydrochloric acids $(\mathrm{HCl})$. When the temperature is further increased the oxides are formed and the extra water is released from the ytterbium hydroxide. Finally, the heat is elevated high enough for the Yb-ion diffusion into silica glass and the sintering of the porous glass.

There are some drawbacks with the solution doping process: the salts have a tendency to self-associate into chemical structures and thus form easily high local concentration variations into the glass. The porosity of the soot layer is also difficult to control, and subsequently the dopant concentrations. MCVD with solution doping is a multi-step, iterative process yielding a doped core of 2-10 layers. The low number of core layers limits the accuracy and flexibility of doping and refractive index profiles. Also the throughput time is fairly large due to multi-step process and this makes the fiber development work slow and expensive.

\subsection{The DND rare-earth doping technology}

With limitations of conventional processes in mind, Liekki Corporation has over the last 6 years focused on commercializing a new active fiber fabrication process meeting the requirements of high power laser applications. The result, DND, represents a paradigm change in active fiber fabrication offering unprecedented doping control and accuracy, extremely high doping, short cycle time, and increased production flexibility. The DND is based on the combustion of gaseous and atomized liquid raw materials in an atmospheric oxy-hydrogen flame ${ }^{11}$. The flexibility in how raw materials are fed to the process gives the freedom of incorporating materials with very different vapor pressures. The glass is doped in-flame where the glass particles are formed, thus the clustering tendency is low. The DND process makes it possible to mix the refractive index changing materials (e.g., alumina, germanium, etc.) with other doping materials (e.g. Yb, Er, Nd, etc.) already during the deposition of the glass particles. This improves the homogeneity of the glass composition prior to the sintering phase. Various parameters affect the formation process, e.g. the vapor pressure of the metals, temperature of the flame, gas velocities, droplet route through the flame and the Gibbs free energy of the raw materials. The qualitative results obtained from the particle size distribution measurement shows a single-peaked particle-size distribution, which indicates that the particles are formed through evaporationcondensation process. Rapid quenching and a short residence time produce small particles with narrow size distribution. Typically the particle size can be adjusted between 10 and $100 \mathrm{~nm}$.

The DND process is depicted in Figure 1. The deposition is a special form of an outside soot deposition process where nanosize particles of doped silica are deposited on the target rod. The deposition process is automated and highly scalable. The deposition results in a highly-uniform soot with 100s of accurately controlled layers. The large number of layers allows accurate control of the radial index difference and active dopant profiles in the fiber core, as shown in the figure 2 where the measured in-fiber $\mathrm{Yb}$ - and Al-ion concentrations are presented for a 30 $\mu \mathrm{m}$ core fiber. Furthermore, very high dopant concentrations with homogeneous glass structure are obtained. 


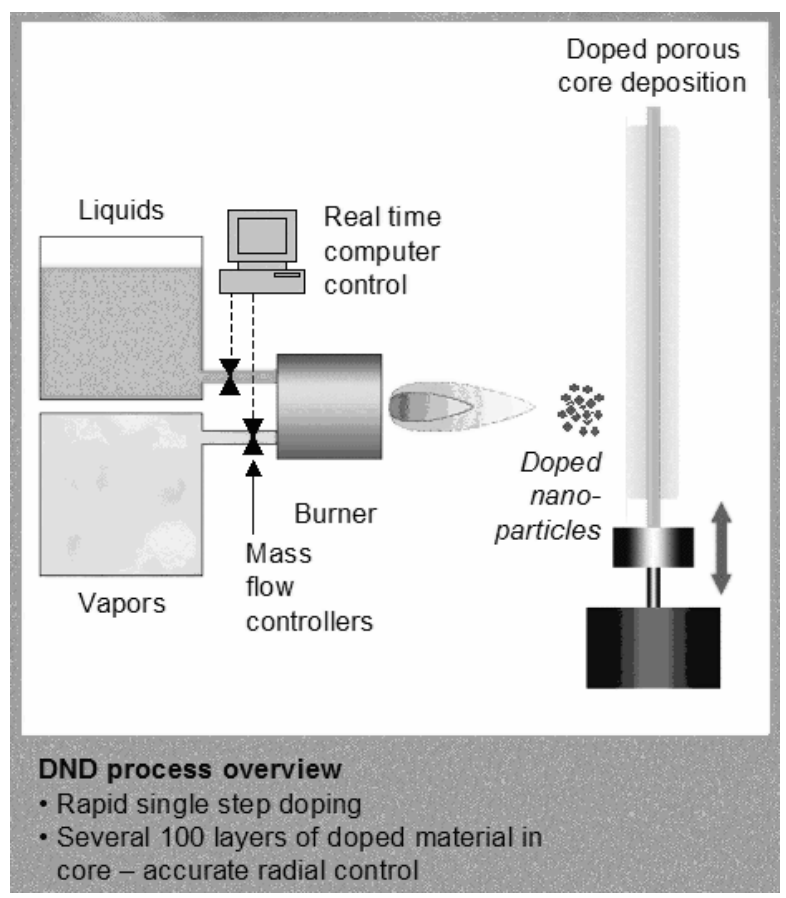

Figure 1: DND process equipment

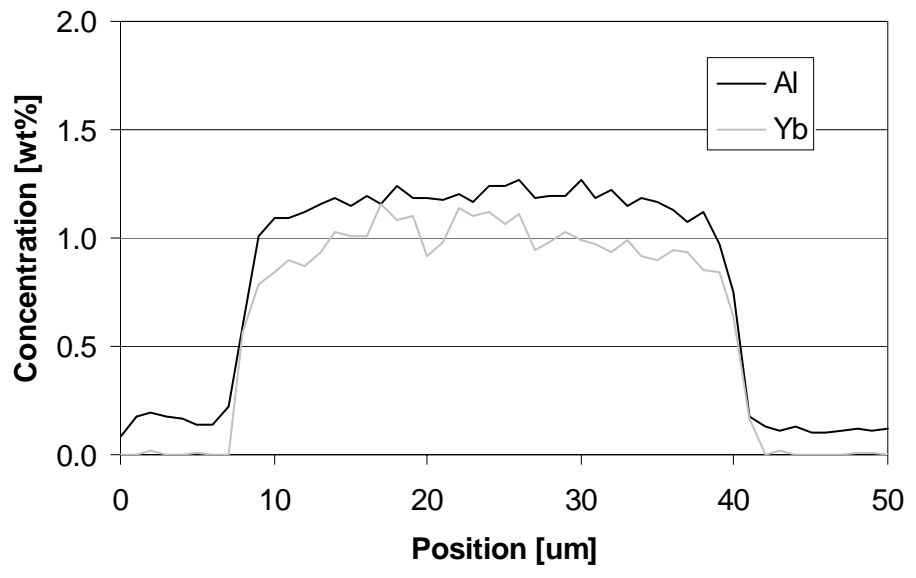

Figure 2: Radial $\mathrm{Yb}$ and Al profiles in the core of the DND fiber.

Sintering phase following the deposition yields solid glass preforms. Typical DND performs are large in diameter and relatively short, simplifying the following glass works and reducing the absolute tolerances. This is especially useful in making large core and large core-to-cladding fibers and PM fibers based on PANDA-design, as well as in making more complex designs such as fibers with rectangular (or other non-circular) cores and claddings, multi-core fibers, or coupled multiple-waveguide fibers. 


\section{MATERIAL}

\subsection{Rare earth local environment}

The controllability of the local environment of the Er ions in the DND process is good enough so that the absorption spectrum shape does not depend on the doping concentration, Figure 3

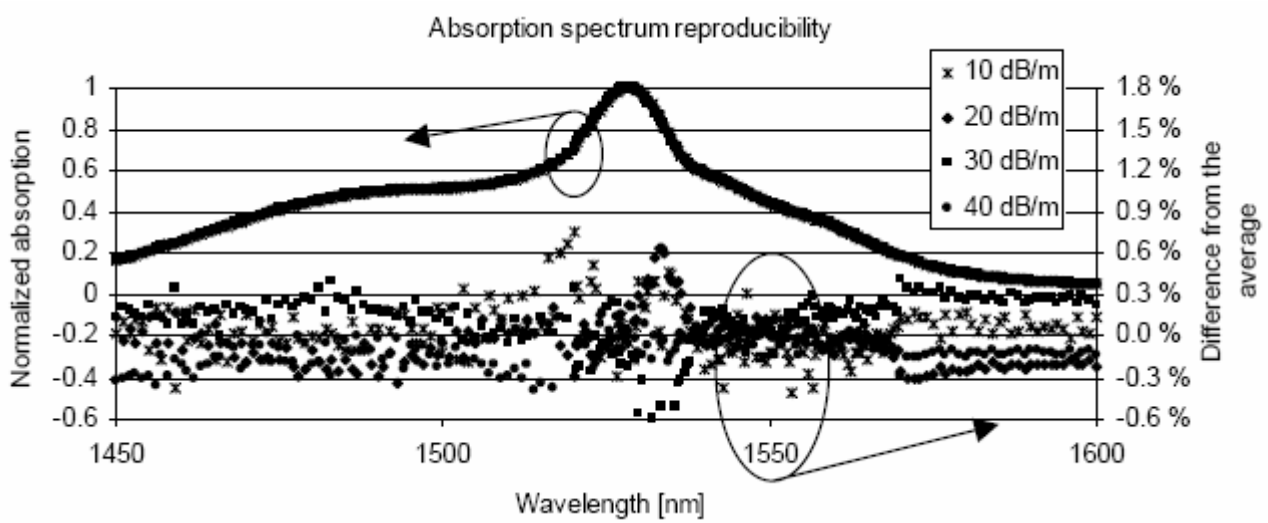

Figure 3: The normalized absorption spectrum of four different Er doped fibers having peak absorptions of 10, 20, $30 \mathrm{and} 40 \mathrm{~dB} / \mathrm{m}$. The possible variation of the spectrum shape is below the measurement accuracy.

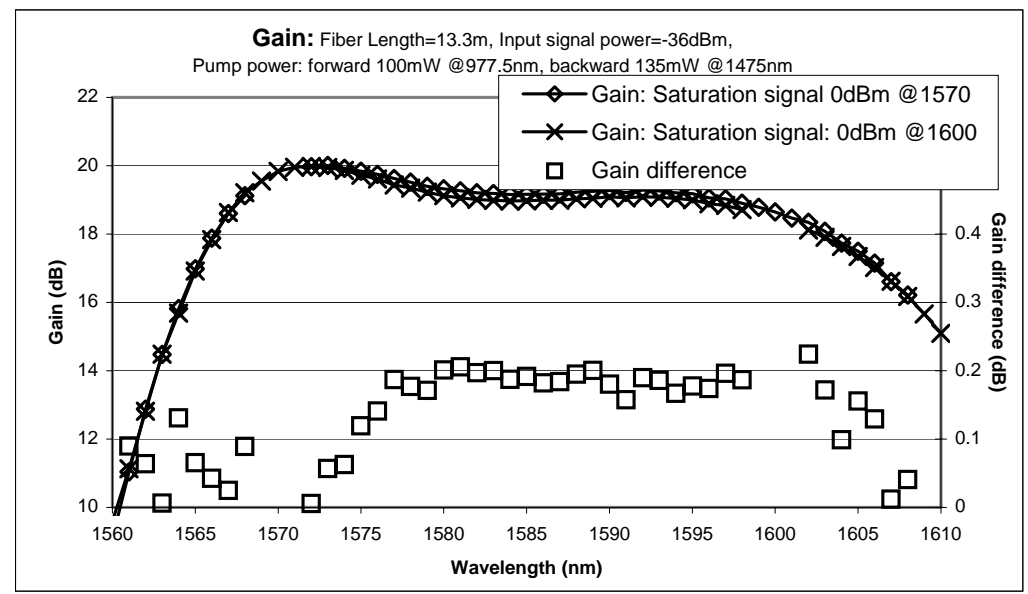

Figure 4: Small signal gain with 0dBm saturating signal and gain difference with two saturating signal wavelengths ${ }^{12}$

The ability to control the local environment of the Er ion also produces broad absorption and emission cross-sections, which can be utilized in L-band amplifiers ${ }^{12}$, lasers ${ }^{13}$ and ultra broadband ASE sources ${ }^{14}$. Due to the uniform doping of the fiber there is almost no spectral hole burning in the L-band amplifier, Figure 4.

\section{PHOTODARKENING IN YB-DOPED SILICA FIBERS}

Photodarkening of rare-earth doped fibers has been reported for many different glasses, doped for example with Tm ${ }^{3+}$, $\mathrm{Ce}^{3+}, \mathrm{Pr}^{3+}, \mathrm{Eu}^{2+}$, and $\mathrm{Yb}^{3+15,16}$. Photodarkening, seen as a temporal increase in the optical loss of the rare-earth doped glass, can be attributed to the formation of color centers in the doped glass core ${ }^{17}$. While most of the excess loss induced by the color centers is at the visible wavelength area, a significant amount of excess loss is present at the signal and pump wavelengths of Yb-fibers. We have observed that for many silica glass compositions the shape of the induced color center is similar, which means that by measuring the excess loss at visible wavelength, like 633nm, we can calculate the excess loss at the signal wavelength ${ }^{18}$.

Photodarkening rate has been measured with inversion levels ranging approximately from $20 \%$ to $60 \%{ }^{19}$. With each higher inversion a faster photodarkening rate was observed, Figure 5 . In log/log scale the linear fit is approximately 7 for both measured fibers ${ }^{19}$. A simple interpretation of this result would be that the photo ionization phenomenon needs 
the energy of seven excited Yb-ions for the creation of a color center. Since the photodarkening process saturates, it can be argued that in addition to the seven excited Yb-ions within energy migration distance also some mechanism to trigger the creation of a color center is needed, for example an impurity. We have also seen that at constant inversion the pump intensity itself does not affect the photodarkening rate ${ }^{18}$.

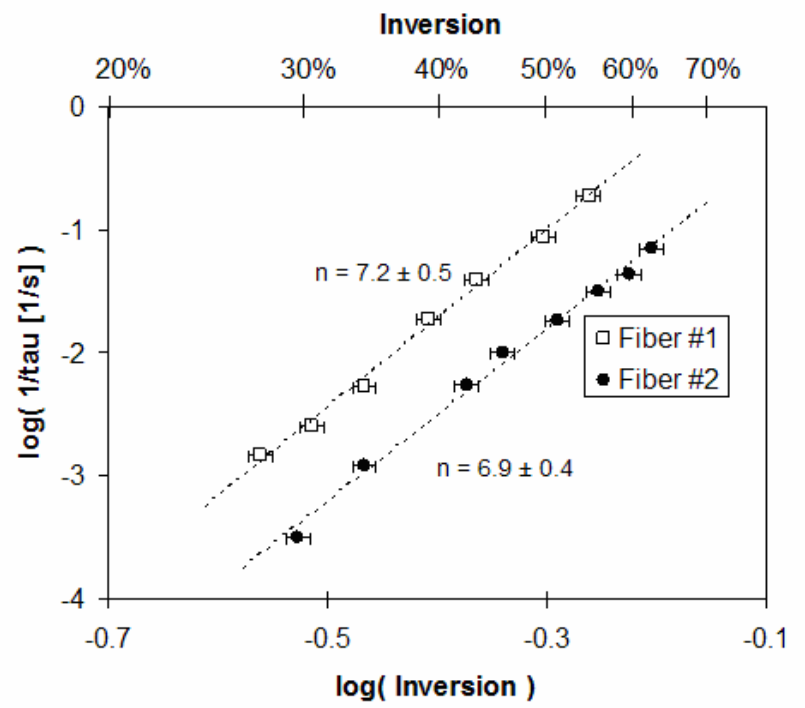

Figure 5: The photodarkening rate has a strong dependence on the inversion, presented here in log-log scale.

Different applications, for example CW lasers and pulsed amplifiers show photodarkening degradation over very dissimilar time scales, depending on the inversion of the fiber. A fiber in a CW fiber laser may have an inversion level of $5-10 \%$, whereas a $40-50 \%$ inversion occurs in a high gain CW or pulse amplifier. Presuming the $7^{\text {th }}$ order inversion dependency of the photodarkening rate, a fiber amplifier may photodarken up to $10^{5}-10^{7}$ times faster than a CW laser. This strong dependence on the inversion must be taken account when characterizing the photodarkening properties of Yb-doped fibers.

The photodarkening depends also on the glass composition. The glass composition changes on the solubility of the rare earth ions and thus has an effect on how the ions are distributed in the glass matrix. As the rare earth ions are more uniformly distributed in the glass, the probability of energy migration between rare earth ions is decreased, and less color centers are generated. The impact of glass composition is illustrated in Figure 6. The figure shows that by changing the glass composition higher resistance to photodarkening is possible even with increased Yb-doping. For a given glass composition the photodarkening rate, and the number of color centers generated increases with higher $\mathrm{Yb}$ concentration. This can be contributed to the increased energy migration probability between Yb-ions.

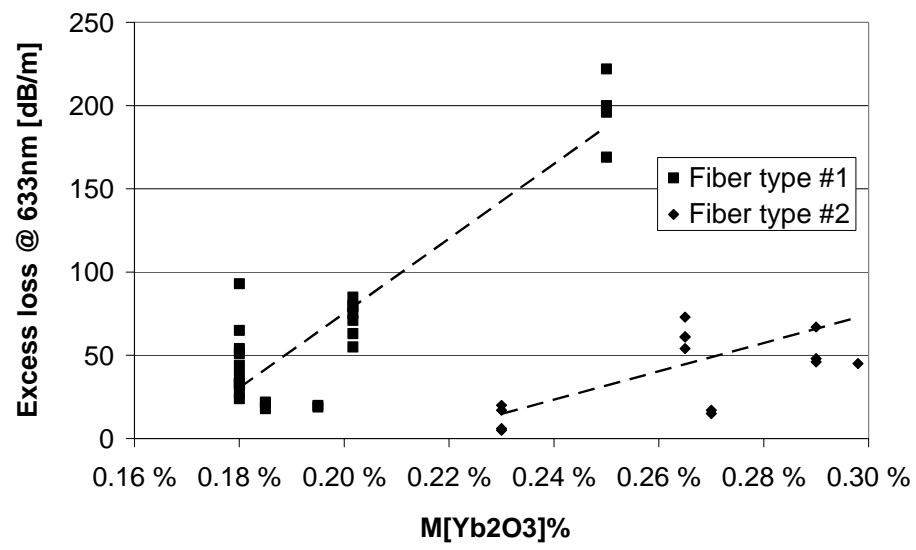

Figure 6: Photodarkening of two different fiber types. Higher doping can be made with lower photodarkening. 


\section{DND FIBERS}

DND technology has been used to develop a large variety of specialty doped fibers. Liekki was one of the first companies in 2002 to introduce highly doped Er fibers with $>12 \mathrm{~dB} / \mathrm{m}$ absorption at 1530nm, enabling much reduced fiber application lengths and thus higher output power before onset on non-linear effects. Furthermore, the highly doped Er fiber from Liekki also opened new possibilities in applications like L-band amplifiers and amplified spontaneous emission (ASE) sources. Liekki Er30-4/125 fiber has been shown to provide $>50 \%$ efficiency in the L-band, giving great advantage in comparison to the lower doped Er fibers used in L-band. Not only the efficiency is better, but through reduced application length also the optical non-linearity and polarization mode dispersion are decreased ${ }^{12}$.

A large number of important applications require high peak powers and pulse energies, which has generated great interest in Yb-doped, large-mode-area fibers. To meet this demand, Liekki has exploited DND technology and aluminosilicate glass to develop a broad range of Yb-doped fibers, ranging from low-power single-mode fibers to $>100 \mu \mathrm{m}$ core double clad fibers with higher than 100W average powers. The common features in the DND-made fibers are high optical-to-optical efficiency, high pump absorption, low photodarkening and excellent beam quality driven by the flat core refractive index and low numerical aperture. As an example, Figure 7 illustrates Liekki Yb1200-20/400DC fiber efficiency and beam quality in a fiber laser configuration.

In pulsed regime, Liekki fiber Yb1200-30/250DC has been used to show that single-stage, mode-filtered, Yb-doped fiber amplifiers are capable of providing diffraction-limited (M2<1.2) output pulses with energies over $1 \mathrm{~mJ}$ and peak powers over $1 \mathrm{MW}$, when seeded with $>20 \mu \mathrm{J}$ pulses $^{7}$. Using a repetition rate of $1 \mathrm{kHz}$, corresponding to complete gain recovery between pulses, these pulse energies and powers were obtained with seed pulse durations of 2.3 ns and 0.38 ns, respectively. The maximum peak power of $1.2 \mathrm{MW}$ generated with the 0.38 ns seed laser corresponds to a peak irradiance of $440 \mathrm{GW} / \mathrm{cm}^{2}$. The maximum pulse energy of $1.1 \mathrm{~mJ}$ generated with the $2.3 \mathrm{~ns}$ seed laser corresponds to a peak fluence of $410 \mathrm{~J} / \mathrm{cm}^{2}$.

Liekki has made a special designed experimental large core double-clad Yb-doped fiber with $80 \mu \mathrm{m}$ core and 400 - $\mu \mathrm{m}$ cladding for multi MW pulsed fiber amplifier applications. A fiber amplifier was built from 3m long piece of this fiber, coiled with a radius of $5 \mathrm{~cm}$ and pumped up to $146 \mathrm{~W} @ 915 \mathrm{~nm}^{8}$. The output beam quality of $\mathrm{M}^{2}=1.2$ and power conversion efficiency of $60 \%$ was achieved. The peak power of the output pulse was $>6 \mathrm{MW}$ when the pulse length was $110 \mathrm{ps}$ and the repetition rate was $50 \mathrm{~Hz}$. When the repetition rate and pulse length was increased to $0.1 \mathrm{MHz}$ and 700 ps respectively, the average output power of $85 \mathrm{~W}$ and peak power over $1 \mathrm{MW}$ was achieved.

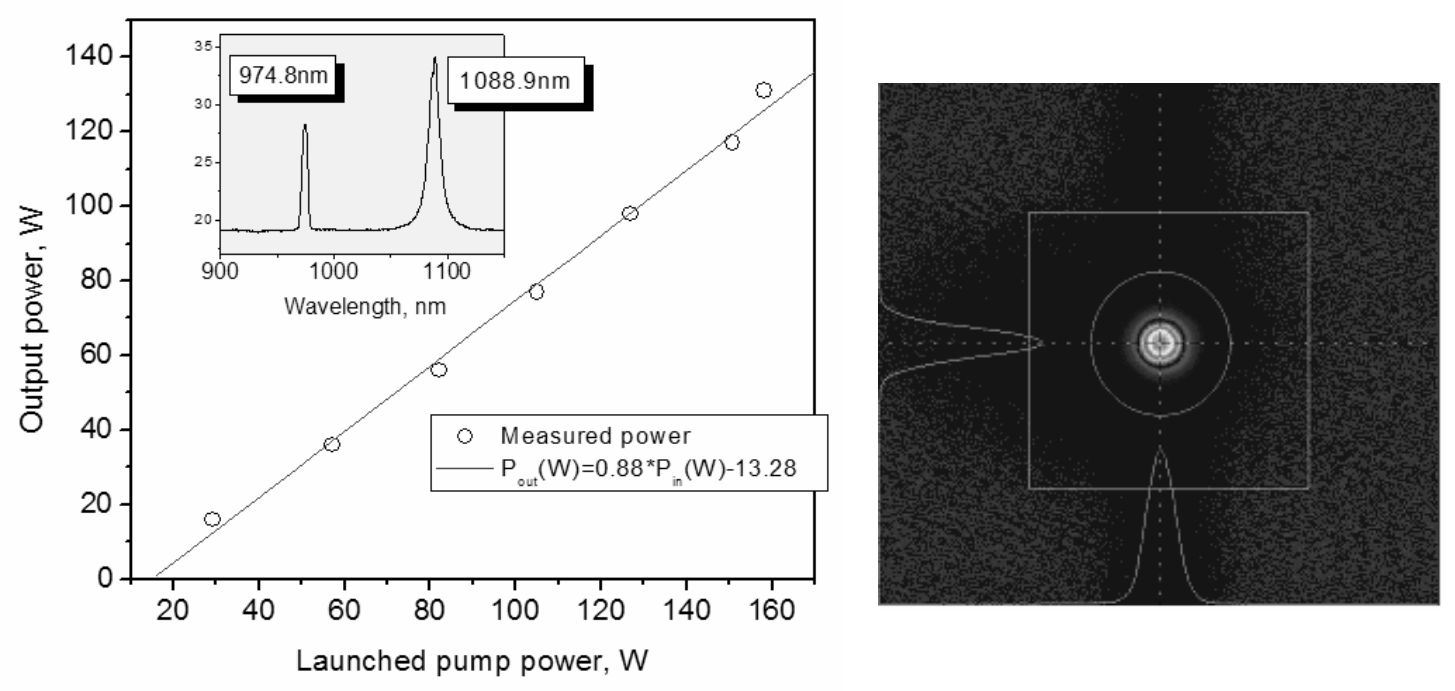

Figure 7: Power conversion efficiency curve of Liekki Yb1200-20/400DC fiber. Inset graph shows the corresponding $\mathrm{M}^{2}$.

The extensive work Liekki has done on measurement and understanding of photodarkening has also revealed that the highly homogeneous DND made core has an advantage in reduced photodarkening in aluminosilicate Yb-doped fibers compared to that of MCDV solution doping, the most prevalent specialty fiber manufacturing technology currently. This difference is highlighted in Figure 8, where the two similar single-mode Yb-doped fibers, one made with DND and the other with MCVD solution doping, are compared. The used measurement method has been developed by Liekki and is described in detail in ${ }^{16}$. 


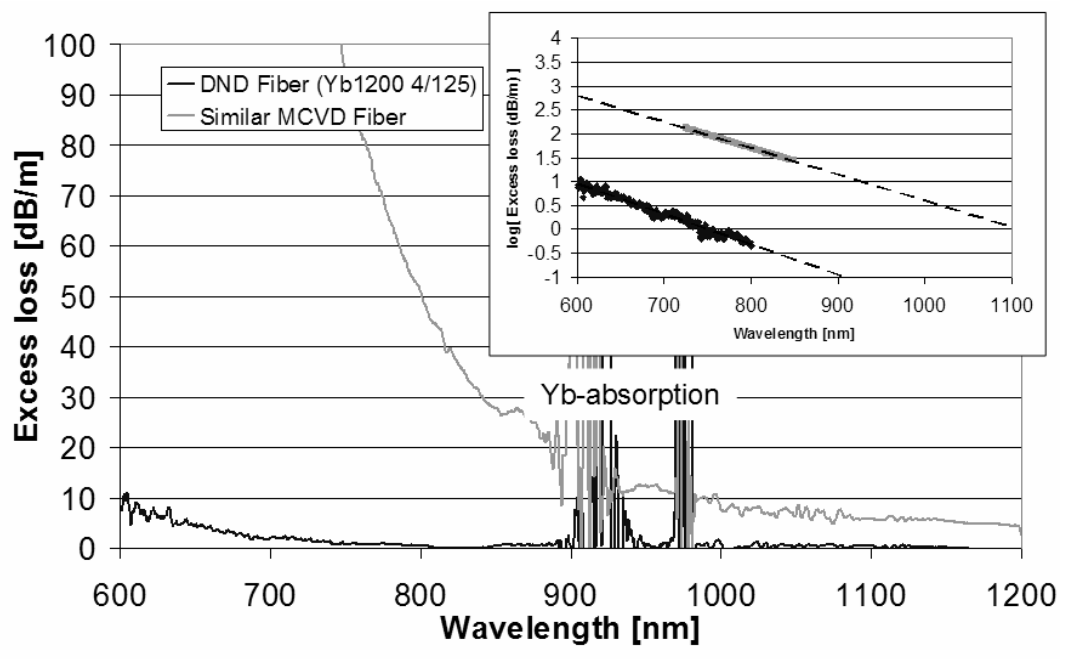

Figure 8: Comparison of DND and MCVD solution doper fiber photodarkening

DND is a rapid and flexible manufacturing process which enables relatively quick prototyping on new core compositions and designs. So far, Yb-ion concentrations ranging from 0.4 to 2 wt \% have been realized with core NA's in the range of 0.05-0.2. In addition to providing excellent optical performance, DND process can produce very large diameter cores, which facilitate the fabrication of large core and large core-to-cladding fibers as well as polarization maintaining fibers. For example, Liekki Yb1200-20/125DC (figure 8 a) fiber features CCRD of 0.16. With 20 $\mu \mathrm{m}$ low NA core and $\sim 1.2 \mathrm{wt} \%$ Yb-ions, the fiber features unpreceded $\sim 27 \mathrm{~dB} / \mathrm{m}$ pump absorption at 976nm in a cladding pumped configuration. Figure 8 b) present Yb1200-30/250DC-PM, polarization maintaining LMA fiber. Even larger cores and core-to-cladding ratios have been manufactured, such as the $80 \mu \mathrm{m}$ core, $400 \mu \mathrm{m}$ cladding fiber shown on Figure 9.

a)

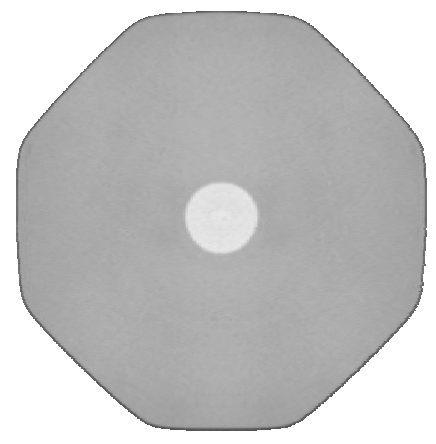

b)

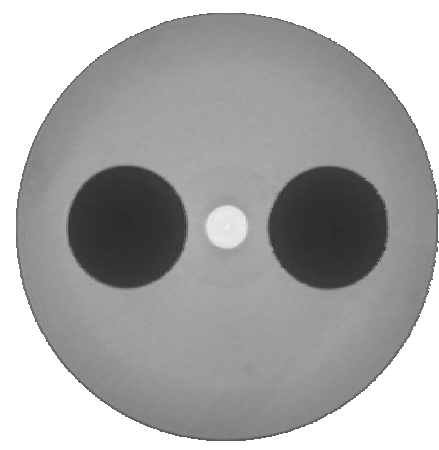

c)

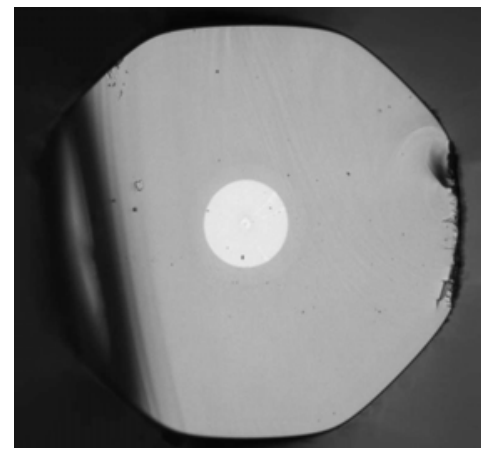

Figure 9: a) Liekki Yb1200-20/125DC b) Yb1200-30/250DC-PM polarization maintaining fiber c) experimental 80 $\mu \mathrm{m}$ core, $400 \mu \mathrm{m}$ cladding DC fiber

\section{CONCLUSION}

Yb-doped double cladding fiber laser systems providing high output beam quality and brightness, multiple mJ pulse energies and MW peak powers require reliable fibers capable of handling high intensity pulses without degrading the beam temporal, spectral or spatial quality. The DND process has been developed with these requirements in mind. DND directly and simultaneously deposits all the elements in nanoparticle size to create preforms in a single step. The endresult is a highly doped core with 100s of layers with accurately controlled composition. More importantly, the doping and the refractive index profile can be independently tailored to produce an optimized active fiber. This ability will further on enlarge the usable mode area of the Yb-doped DC fibers providing practical solutions for the high (peak) power fiber laser industry. A further advantage of DND is the ability to mitigate photodarkening due to flexible engineering of the glass composition and the excellent homogeneity of the resultant glass. 
The rapid development of fiber laser applications is setting new requirements for active fibers. A new active fiber fabrication technology, that can produce the next generation active fibers required, may therefore be called for. We believe Direct Nanoparticle Deposition technology offers a solution, and that it represents a paradigm change in active fiber fabrication offering unprecedented doping control and accuracy, extremely high doping, short cycle time, and increased production flexibility.

\section{ACKNOWLEDGEMENT}

We acknowledge helpful discussions with Dahv Kliner, Jeff Koplow, and their group at Sandia National Laboratories. 


\section{REFERENCES}

1. H. Po, E. Snitzer, R. Tumminelli, L. Zenteno, F. Hakimi, N. M. Cho, and T. Haw, "Double clad high brightness Nd fiber laser pumped by GaAlAs phased array”, Optical Fiber Communications Conference Technical Digest, PD7 (1989)

2. J. Nilsson et al., "High power fiber lasers”, Optical Fiber Communications Conference technical digest, OTuF1 (2005)

3. C.-H. Liu, B. Ehlers, F. Doerfel, S. Heinemann, A. Carter, K. Tankala, J. Farroni, and A. Galvanauskas, "810W continuous-wave and single transverse-mode fibre laser using $20 \mu \mathrm{m}$ core Yb-doped double-clad fibre”, Electronics Letters 40, 23 (2004)

4. R. Paschotta, J. Nilsson, A. C. Tropper, and D. C. Hanna, “Ytterbium-Doped Fiber Amplifiers”, IEEE Journal of Quantum Electronics 33, 7 (1997)

5. N.S. Platonov, D.V. Gapontsev, V.P. Gapontsev, V. Shumilin, “135W CW fiber laser with perfect single mode output”, Lasers and Electro-Optics, CLEO Technical Digest, CPDC3 (2002)

6. S. Norman, M. Zervas, A. Appleyard, M. Durkin, R. Horley, M. Varnham, J. Nilsson, Y. Jeong, "Latest development of high power fiber lasers in SPI”, in Fiber Lasers: Technology, Systems, and Applications, L.N. Durvasula, ed., Proc. SPIE 5335, 229-237 (2004)

7. R. L. Farrow, D. A. V. Kliner, P. E. Schrader, A. A. Hoops, S. W. Moore, G. R. Hadley, and R. L. Schmitt, "Highpeak-power (>1.2 MW) pulsed fiber amplifier”, will be presented in PW06 LASE conference, paper 6102-22 (2006)

8. K.C. Hou, M.Y. Cheng, A. Galvanauskas, D. Engin, R. Changkakoti, and P. Mamidipudi, "Multi-MW Peak Power Scaling of Single-Transverse Mode Pulses using 80- $\mu \mathrm{m}$ Core Yb-doped LMA Fibers", will be presented in PW06 LASE conference, paper LBD 2 (2006)

9. S. Sudo, Optical Fiber Amplifiers: Materials, Devices, and Applications, Chap. 2.6, Artech House, Inc., Boston (1997)

10. B.J. Ainslie, “A review of the fabrication and properties of erbium-doped fibers for optical amplifiers,” Journal Of Lightwave Technology 9, 220-227 (1991).

11. S. Tammela et al., "Direct Nanoparticle Deposition process for manufacturing very short high gain Er-doped silica glass fibers,” ECOC’02, ECOC 2002 Proceedings, 4, 9.4.1-9.4.3 (2002)

12. S. Tammela et al., "Very short Er-doped silica glass fiber for L-band amplifiers," OFC’03, OFC2003 Technical digest, 1, 376-377 (2003)

13. L. A. Gomes et al., "140-MHz stretched pulse ytterbium fiber laser operating in the 980-1030 nm spectral range," OSA Annual Meeting - New Frontiers in Optics, 5-9 October, Tucson, Arizona, USA (2003)

14. S. Tammela et al., " Potential of nanoparticle technologies for next generation erbium-doped fibers” OFC’04, OFC2004 Technical digest, FB5 (2004)

15. M. M. Broer, D. M. Krol, and D. J. DiGiovanni, "Highly nonlinear near-resonant photodarkening in a thuliumdoped aluminosilicate glass fiber”, Optics Letters 18, 10 (1993)

16. J. Koponen, M. Söderlund, S. Tammela, and H. Po, “Measuring photodarkening from Yb-doped fibers,” in Proceedings of CLEO/Europe ’05, CP2-2-THU (2005)

17. L. B. Glebov, "Linear and Nonlinear Photoionization of Silicate Glasses”, Glass Science and Technology 75, C2 (2002)

18. J. Koponen, M. Söderlund, S. Tammela, and H. Po, ”Photodarkening in ytterbium-doped silica fibers”, in Optically Based Materials and Optically Based Biological and Chemical Sensing for Defense II, J. C. Carrano, A. Zukauskas, A. W. Vere, J. G. Grote, F. Kajzar, Proc. SPIE 5990, 72 (2005)

19. J. Koponen, M. Söderlund, S. Tammela, D. Kliner, and J. Koplow, "Photodarkening rate in ytterbium doped silica fibers”, will be presented in PW06 LASE conference, paper LBD 3 (2006) 